
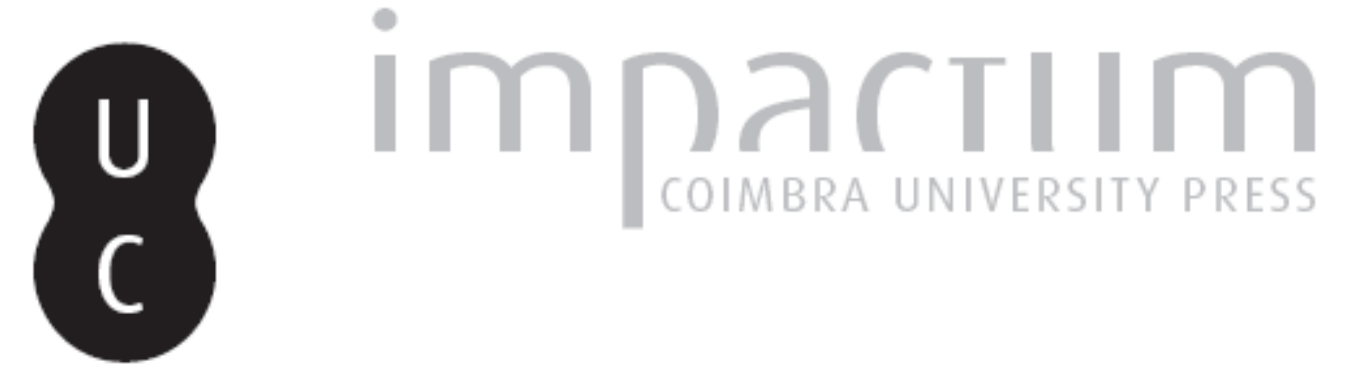

\title{
Les Carnets de Captivité, pardelà la mort, une ouverture sur le visage de Levinas
}

\section{Autor(es): $\quad$ David, Alain; Bernardo, Fernanda}
Publicado por: Faculdade de Letras da Universidade de Coimbra, Instituto de Estudos Filosóficos

URL persistente:

URI:http://hdl.handle.net/10316.2/29465

DOI:

DOI:http://dx.doi.org/10.14195/0872-0851_41_5

Accessed : $\quad$ 26-Apr-2023 11:03:32

A navegação consulta e descarregamento dos títulos inseridos nas Bibliotecas Digitais UC Digitalis, UC Pombalina e UC Impactum, pressupõem a aceitação plena e sem reservas dos Termos e Condições de Uso destas Bibliotecas Digitais, disponíveis em https://digitalis.uc.pt/pt-pt/termos.

Conforme exposto nos referidos Termos e Condições de Uso, o descarregamento de títulos de acesso restrito requer uma licença válida de autorização devendo o utilizador aceder ao(s) documento(s) a partir de um endereço de IP da instituição detentora da supramencionada licença.

Ao utilizador é apenas permitido o descarregamento para uso pessoal, pelo que o emprego do(s) título(s) descarregado(s) para outro fim, designadamente comercial, carece de autorização do respetivo autor ou editor da obra.

Na medida em que todas as obras da UC Digitalis se encontram protegidas pelo Código do Direito de Autor e Direitos Conexos e demais legislação aplicável, toda a cópia, parcial ou total, deste documento, nos casos em que é legalmente admitida, deverá conter ou fazer-se acompanhar por este aviso.

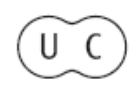




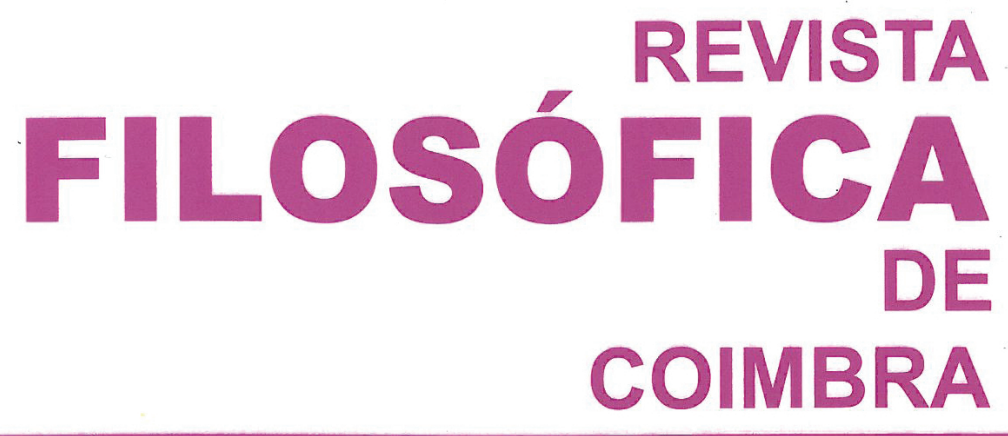

vol. 21 - número 41 - março 2012

vol. 21 - número 41 - março 2012

Fundação Eng. António de Almeida

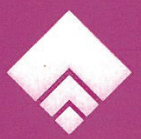




\title{
Les Carnets de Captivité, par-delà la mort, une ouverture sur le visage de Levinas
}

\author{
Alain David e Fernanda Bernardo*
}

Fernanda Bernardo: La publication des CARNETS DE CAPTIVITÉ ET AUTRES INÉDITS (Editions Grasset et Fasquelle /IMEC Editeur) - le premier volume des æeuvres complètes d'Emmanuel Levinas - à l'automne 2009, sous la responsabilité de Rodolphe Calin et de Catherine Chalier, fut sans doute un événement éditorial et philosophique majeur.

Quel fut pour toi, Alain David, qui, dans ta situation de lecteur et d'ancien participant au séminaire de Levinas à la Sorbonne, connaissais bien sa pensée philosophique, la portée de cet événement? Ou ta surprise, s'il y en a eu une pour toi?

Alain David: D'abord beaucoup d'émotion. Comme tu as la gentillesse de le rappeler j'ai assisté au séminaire et aux cours de Levinas de 1973 jusqu'au début des années 80, où il mit fin à son enseignement. En 1973 je faisais mon service militaire, et j'avais obtenu du colonel commandant mon unité à Saint-Dizier l'autorisation d'aller suivre une journée par semaine les cours de la Sorbonne. J'avais fait une maîtrise à Dijon, sans avoir le

\footnotetext{
* Oralmente e por escrito, entre Coimbra e Paris, este diálogo em torno dos Carnets de Captivité et autres inédits de Emmanuel Levinas (Editions Grasset et Fasquelle/IMEC Ed., Paris, 2009) decorreu de Janeiro de 2011 a Janeiro de 2012 e eu expresso aqui o meu reconhecimento a Alain David pelo prazer desta partilha admirativa e sempre amigável do pensamento deste filósogo que ambos temos por um «Mestre» - Alain David que MarieAnne Lescourret tem pelo «filho pródigo» de Levinas e que eu não direi um «levinasiano» de excepção - convicta, que sou, de que levinasiano só mesmo Levinas o podia ser! - mas um admirável exemplo vivo do pensamento de Emmanuel Levinas. Um pensamento urgentemente necessário na sua quase-impossível exigência na perigosa barbárie da civilização do inter-esse que sombriamente desenha o horizonte dos nossos dias.
} 
sentiment d'être déjà rentré en philosophie, sans savoir ce que je cherchais, sans être même capable de lire les grands textes. La rencontre de Levinas fut pour moi une révélation et un bouleversement, c'est à travers lui que je pus enfin avoir accès à ce que signifie la philosophie, et cette situation perdure aujourd'hui. C'est te dire que ce volume paru en 2009 m'arrive comme une grâce, du lointain de ma jeunesse, dans un sentiment quasi proustien. Peut-être parce que le caractère inorganisé de ces inédits me replace, davantage que ne l'aurait fait un texte construit, devant ce qui était la personne vivante de Levinas, son rire et son sourire, son accent, sa façon de parler, sa manière de se tourner vers un interlocuteur lorsqu'il faisait cours, de lever l'index, voire les deux index, appuyé en arrière sur le dossier de sa chaise, pour solenniser une déclaration... Y a-t-il eu surprise ? Oui, certainement. Non que cette publication en elle-même eût été inattendue, je savais qu'elle se préparait, au milieu de difficultés, de circonstances qui l'auraient sans doute infiniment peiné. Mais surprise, néanmoins, au sens où pour Bergson le réel vient toujours surprendre le possible, au sens de la merveille : « le visage parle ». Ce livre représente, par-delà la mort, une ouverture, sur le visage de Levinas.

Fernanda Bernardo: Bien que très rarement, et toujours d'une façon retenue à l'extrême, de l'expérience des années 40, Emmanuel Levinas avait lui-même déjà parlé dans son ouvre philosophique comme celle d'une interruption de sa vie, dans sa vie, comme celle d'un "gouffre béant » que rien ne pouvait combler ni même recouvrir, et dont il porterait à jamais la " tumeur dans sa mémoire ». Je pense ici à «Sans Nom» (1966) et à «Signature», notamment, pour ne rien dire des dédicaces d'AUTREMENT QU'ÊTRE OU AU-DELÀ DE L'ESSENCE (1974).

Les CARNETS DE CAPTIVITÉ (1940-1945) inscrivant en eux la mémoire du jour le jour de cette expérience datée, dont ils sont le témoignage émouvant, comment vois-tu maintenant cette même expérience s'inciser et se marquer à nouveaux frais dans la pensée à l'œuvre dans l'œuvre d'Emmanuel Levinas? Qu'apportent-ils d'autre, ces CARNETS, à la singularité, voire à l'idiomaticité de la pensée philosophique de Levinas? Ou, comment la re-marquent-ils davantage? Éclairent-ils davantage sa "genèse», son «orientation» et son «timbre» méta-éthiques?

Alain David : Effectivement Levinas dans «Signature », le fragment autobiographique inséré à la fin de Difficile liberté, présente sa vie et son œuvre comme conditionnées par le « pressentiment et le souvenir de l'horreur nazie » : pressentiment, souvenir, termes qui arrivent comme une 
reprise de la rétention et de la protention husserliennes. "Pressentiment 》 est d'ailleurs lui aussi un terme husserlien (Vorahnung ist der gefühlmässige Wegweiser für alle Entdeckungen. Le pressentiment est, dans tous les ordres de découverte, le détecteur affectif - trad. Ricoeur de la Conférence de Vienne) Mais ici le pressentiment est le pressentiment de ce pour quoi " les noms nous manquent ». Il n'ouvre sur aucun présent - manque au présent dont Levinas a fait un moment central de sa philosophie - et dont atteste encore un texte comme «Sans nom ». Faut-il voir alors dans les Carnets un document sur l'expérience concentrationnaire, comparable en quelque sorte à Si c'est un homme de Primo Levi ? Evidemment non : parce que déjà Levinas n'a pas vécu l'expérience concentrationnaire, aussi dure qu'ait été sa captivité ; parce que de cette dureté elle-même on trouve très peu d'écho dans les Carnets. Parce que également, si j'ose ici apporter mon modeste témoignage, je n'ai jamais entendu Levinas évoquer l'événement de l'extermination alors qu'on commençait à Paris ( notamment depuis la reprise par Lyotard en 1980, au premier colloque Derrida de Cerisy, de la formule d'Adorno « que signifie penser après Auschwitz ») à faire de cette évocation un moment obligé de la pensée. L'expression " horreur nazie » reste très sobre, je ne sais pas comment Levinas aurait réagi à celle de Shoah, qui à la fin des années quatre-vingts n'était pas encore très employée. Jacques Rolland m'a raconté que Levinas se refusait à prononcer le nom d'Auschwitz. Alors, à cet égard, de quoi est-il question dans les Carnets? Allusivement, seulement de son expérience quotidienne de prisonnier, et jamais sur le mode de la plainte. En revanche, beaucoup, de ses préoccupations intellectuelles, de son travail de philosophe. Là encore on pourrait penser à Husserl, qui dans la Conférence de Vienne, justement, n'évoque les bouleversements que connaît l'Europe et qui l'ont pourtant frappés très directement, que sur le mode d'une crise de la raison et de son espérance placée dans la phénoménologie. Je vois pourtant une différence importante, et qui constitue sans doute le noyau de sa lecture de Husserl : le pressentiment est, chez Levinas, le rapport à l'absence et non l'ouverture sur le plein d'un présent. Rapport à l'absence au sens de ce qui n'a jamais été présent. Rapport qu'il a pu décrire par ailleurs comme le " pré-philosophique » que représente la littérature, laquelle est sans arrêt mentionnée dans les Carnets. Un préphilosophique qui s'entrelace donc à la philosophie, qui s'unit à elle " tératologiquement» (Humanisme de l'autre homme), qui constitue le fil directeur de son rapport à la philosophie. Tu me demandes ce qui m'a surpris, et ce que les Carnets apportent de nouveau qui permette d'entendre la singularité et l'idiomaticité de la pensée de Levinas. Outre le caractère en lui-même passionnant de work in progress, la nouveauté est à mon sens (ce n'est d'ailleurs pas contradictoire) la place de la littérature, 
qui ici n'est pas un à-côté de la philosophie, mais la philosophie elle-même ; et ainsi la vocation d'écrivain de Levinas ; l'omniprésence d'auteurs que le lecteur attentif de Levinas pouvait deviner, mais qui ici est attestée (Proust, notamment) ; d'une certaine façon la mise en récit de l'événement même de la guerre et de son expérience de prisonnier. Tout cela est passionnant à mes yeux, parce que confirmant des intuitions et des hypothèses de lecture que l'on pouvait timidement avoir, induisant pour l'avenir tout un travail de lecture qui mettrait l'accent, bien davantage, par exemple, que sur la concurrence entre la place de Heidegger et celle du judaïsme, sur l'écriture de Levinas.

Fernanda Bernardo: Pour continuer avec la question de savoir comment pour toi les "Carnets» éclairent le rapport de Levinas à la captivité et celui de celle-ci à sa pensée et à son œuvre, peux-tu, Alain, préciser un peu ce que tu entends par "la place de la littérature, qui ici n'est pas un à-côté de la philosophie, mais la philosophie elle-même; et ainsi la vocation d'écrivain de Levinas ": tout cela que tu présentes comme étant l'apport majeur des CARNETS à la compréhension de l'idiome éthico-philosophique de Levinas.

Je précise ma question : "la littérature» - sûrement pas au sens des Belles Lettres! - serait-elle alors ici un autre nom pour la philosophie? Pour l'éthique comme philosophie première, plutôt ? "La littérature», aurait-elle donc ainsi aux "Carnets» la même radicalité, la même matinalité insomniaque et la même portée que l'éthique au sens lévinassien, c'est-à-dire comprise dans un registre méta-philosophique, méta-ontologique, méta-gnoséologique, et même méta-éthique?

Car nous connaissions déjà toute l'importance de "la littérature» pour Levinas - à François Poirié et à Myriam Anissimov, notamment, il avait même avoué qu'il avait été sa lecture des romans russes (Pouchkine, Lermontov, Tolstoï, Dostoïevski, ...), dans sa jeunesse, qui l'avait emmené à l'étude de la philosophie. Et on n'oubliera pas non plus que le tout premier mot dont, en 1935, Lévinas a fait comme un philosophème pour traduire le besoin de sortir de l'être, et donc comme la toute première catégorie philosophique pour suggérer la nécessité de l'autre voie que celle de l'être, a été celui d'évasion : un mot que Levinas confesse avoir emprunté au langage de «la littérature» et de la critique littéraire de son temps, justement.

Cela dit, je me demande : en lisant les «Carnets», et notamment les annotations et les citations de la lecture de Levinas des écrivains qui l'accompagnaient en captivité (Tolstoï, Dostoïevski, Proust, Baudelaire, Bloy, Shakespeare, ...), ne reste-t-on pas aussi avec l'impression que «la 
littérature»-de même que l'épreuve de sa captivité - lui aura également inspirée l'orientation éthico-métaphysique qui viendra à aimanter et à timbrer sa pensée philosophique? Je m'explique : sans oublier qu'au moment de sa captivité Emmanuel Levinas était déjà l'auteur de QuelQues RÉFLEXIONS SUR LA PHILOSOPHIE DE L'HITLÉRISME (1934) et de DE L'ÉVASION (1935), notamment et pour l'essentiel en ce qui concerne ici ma question, la lecture des "Carnets» ne nous suggère-t-elle pas que «la littérature» aura, elle aussi, inspirée à Levinas le tournant éthico-métaphysique de sa pensée en lui dévoilant la direction qu'il se cherchait depuis DE L'ÉVASION (1935), au moins, pour sortir de l'être? Je pense notamment ici aux notes de sa lecture de Tolstoï et de Dostoïevski - des notes qui soulignent leur "passion de chaque être» et leur quête de "la nudité», (cf. "Carnet» 2, p. 87 ; "Carnet $3 »$, p. 100). Et je pense aussi et surtout aux annotations de la lecture de Levinas de Proust - qu'il tenait par «le poète du social», «du social pur»- dont il dit au «Carnet $2 »$, daté de 1942, que "Toute l'histoire d'Albertine prisonnière - est l'histoire de la relation avec autrui.» Tandis que dans une annotation du "Carnet 7», datée de 1946, Levinas écrit : "Ma philosophie - est une philosophie du face-à-face. Relation avec autrui, sans intermédiaire.» C'est déjà là, on le sait bien, comme une définition de son éthique.

Cela dit, je poserai autrement ma demande: ce tournant d'une philosophie de l'évasion de l'être (1935) vers une philosophie de l'autrement qu'être, du vers l'autre sans condition, n'a-telle pas aussi implicite, ne dégage-t-elle pas également déjà une toute autre conception de «la littérature»? Une attention à sa condition de possibilité - plutôt que la seule attention au registre pré-philosophique et ontologique que, selon Levinas, est bien aussi celui des littératures et des livres qui nous portent et nous soutiennent? Bref : "la littérature» ne serait-elle pas aussi un autre nom du "vers l'autre»? Ce tournant n'est-il pas aussi à l'origine de ce que, plus tard, Levinas nommera «la littérature avant la lettre», ou bien alors "la littérature de la littérature»? N'est-ce pas déjà cette «littérature de la littérature», j'oserai même dire, n'est-ce pas déjà cette portée éthico-métaphysique de "la littérature qui émerge déjà des annotations de Levinas sur les écrivains dans les «Carnets»?

Alain David : Je sais que je vais manquer des choses par rapport à ta question tellement riche, et, de surcroît, redoutablement complexe, et j'en demande d'avance pardon. Je commencerai par le mot principal de tout cela : la littérature ; comprise entre un sens minimal, les « Belles Lettres ", et un sens maximal, la philosophie, ou même, comme tu le dis, le méta..., méta-philosophique, méta-ontologique, méta-gnoséologique, 
méta-éthique. Pour commencer, je ne suis pas sûr qu'il faille exclure les «Belles Lettres », peut-être pas davantage qu'il ne faut retirer le « bel canto » de la musique. Ou pour prendre une référence plus porteuse sans doute, est-ce que la littérature pour Levinas est équivalente à la Dichtung pour Heidegger ? Cette dernière n'est pas simplement la «poésie », elle est la parole parlante (die Sprache spricht) dont la profondeur s'égale à la hauteur de la pensée, et dont l'exemple essentiel est donné par l'œuvre de Hölderlin. Il ne s'agit donc pour Heidegger nullement de versification ou de prosodie. Est-ce que Levinas avait la même réserve aristocratique vis-à-vis de la littérature ? Je ne le crois pas : à preuve, les exemples qu'il choisissait. C'étaient souvent les textes les plus classiques du patrimoine littéraire, Corneille et Racine, pour la France, comme il lui arrivait de citer en peinture « la Joconde »- j'oserai ajouter, comme Kant pouvait prendre la musique militaire comme exemple pour la musique. Cela veut dire d'abord que Levinas appréciait la littérature sans se poser en expert, sans rechercher les œuvres ésotériques (si l'on excepte l'œuvre littéraire de Blanchot). Il n'a pas commenté Mallarmé, par exemple, et s'il lui arrivait de citer, c'est à la manière des " pages roses du Larousse » (expression qu'il aimait d'ailleurs employer) par exemple le « profond jadis, jadis jamais assez » de Valéry, ou le «messire Gaster » de Rabelais, ce qui ne l'a jamais conduit à un commentaire du "Cantique des colonnes », de Gargantua ou du Tiers livre. Autrement dit la littérature affleure dans sa pensée comme ces phrases résiduelles de la langue, de celles que l'on cite dans les salons ou les dissertations, pour attester de sa culture, mais qui par là même attestent de surcroît, à l'insu de ceux qui s'y réfèrent, que tout le discours n'est qu'un acte manqué de la langue dont le sens est de faire penser à la vérité des œuvres. Et cette dernière ne suppose pas une solennisation particulière, comme la Dichtung heideggerienne, elle est constituée de toutes les œuvres, les petites ou les grandes, éventuellement les plus « kitsch », le mauvais goût même, ainsi ce que Adorno pouvait mentionner à propos de la musique de Mahler, s'extasiant devant le caractère citationnel du tintamarre ambiant. Comme je le comprends, c'est par là que les « littératures nationales sont bibles », par le fait qu'en elles est attestée la sainteté de la langue, qui n'est pas le dévoilement de la sacralité de l'être, mais le l'un-pour-l'autre, la sainteté de l'expression.

Littérature et éthique : oui. Tu le sais bien, Derrida y a souvent insisté, Levinas a finalement hésité devant le mot d'éthique, pour le remplacer par «sainteté » (l'article du Figaro que j'ai mentionné tout à l'heure portait comme titre «j'appelle éthique la sainteté possible »). Le mot sainteté est moins grec dans son origine, mais peu importe à mon sens : il s'agit rarement chez Levinas d'étymologie. Ce qui est intéressant avec 
sainteté est que ce mot est au plus proche et au plus loin de sacralité, et que là se précise un enjeu qui non seulement modalise tout le sens de la confrontation à Heidegger, mais encore et surtout, indépendamment de Heidegger, tout le sens de l'engagement que constitue l'œuvre de Levinas : un engagement contre le sacré, cet engagement étant la condition de « la sainteté possible ». La littérature est sainte, elle dit pour les nations, dans les multiples langues des nations, et de toutes les manières possibles, la signification du l'un-pour-l'autre, l'unicité de Dieu. Je voudrais rappeler la dernière phrase de "Langage et proximité », ce texte extraordinaire, jamais repris par la suite, mais certainement l'une des inspirations de Autrement qu'être : «Ce premier dire n'est certes qu'un mot. Mais c'est Dieu ». Ce premier Dire, inhérent à tout dire comme son kérygme même, est la littérature.

Je voudrais enfin ajouter quelque chose à propos de ton évocation de l'éveil, de la sortie de l'horreur de l'être : j'ai là-dessus une opinion assez radicale, différente de celle de la plupart des commentateurs, qui voient (textes à l'appui, je le reconnais) dans la pensée de l'être non seulement la trace de la référence à Heidegger, mais également comme une première étape de la pensée de Levinas, étape dépassée ensuite par Autrement qu'être. Il me semble au contraire que la pensée de l'être a toujours été mise du côté de ce qu'il fallait dénoncer, de ce qui était pour l'humanité une impasse, une erreur ou une faute, une mauvaise fascination, associable à l'ensorcellement : en cela - et je sais bien que je durcis les oppositions, que j'utilise des mots qui ne sont pas ceux de Levinas - j'évoquerai l'illusion transcendantale kantienne - ce qui renvoie à l'hypothèse d'un kantisme fondamental de Levinas, hypothèse que je serais pour ma part, prêt à défendre.

Fernanda Bernardo: Ce que tu viens de dire suscite chez moi deux questions, ou deux perplexités. Voici d'abord la première : j'avoue avoir du mal à te suivre quant à cette "équivalence " que tu décrètes entre la " littérature " chez Levinas et la "Dichtung » chez Heidegger, laquelle, dis-tu, n'est pas simplement la " poésie » mais la "parole parlante 》 (die Sprache spricht). Peux-tu préciser ce que tu entends là par "équivalente». "Equivalente " quant à leur radicalité ? Quant à la radicalité qu'elles signifient, en comprenant par là la condition de possibilité de ce que Levinas appellera la " signification de la signification ", "le sens du sens 》, ou "le sens des sens 》, voire "l'œuvre "? Radicalité qui, de toute façon n'est pas la même chez les deux philosophes, car elle passe par la différence entre la transcendance de l'autre, du tout autre de Levinas et pour Levinas, voire la différence "éthico-métaphysique ", 
et celle, ontologique, de l'être pour Heidegger, "le transcendens pur et simple » dans le dire de SEIN UND ZEIT. Et justement, en raison même de l'hyperbolicité de sa pensée dictée et aimantée par l'autrement qu'être ou au-delà de l'essence, Levinas n'a pas de mots assez durs envers la fameuse "langue qui parle", de Heidegger, et qui "instaure le monde dans l'être ", car, langage pré-logique et pré-syntaxique, certes, mais de toute façon langage du dévoilement, elle est, dit-il, la langue du neutre, de l'anonymat, de personne et pas celle du "me voici » ou de l'extravagance du Dire. Celle de l' " adieu », finalement. C'est là la raison pour laquelle, par contre, je te suis absolument quand tu dis du Dire - le "premier Dire », le "Dire " étant toujours premier malgré sa répétition - qu'il " est la littérature ». Mais, justement, n'est-ce pas parce que la littérature est cela même chez Levinas, voire le salut à l'autre avant la langue, dans la langue même - une langue toujours adressée - comme un instant d'aphasie, bref comme un clin d'œil à l'autre, un Wink, qu'elle n'est pas, pas seulement et pas d'abord en tout cas, les "Belles Lettres»?

Alain David: Il me semble que tu as absolument raison sur le terme que j'ai utilisé d' " équivalence » : la littérature et la Dichtung ce n'est pas du tout " pareil ». Non seulement ce n'est pas identique (gleich) comme aurait dit Heidegger), mais ce n'est pas non plus « le même », das Selbe. Mais derrière ce que tu as raison de pointer comme une imprudence verbale de ma part, il y avait sans doute la volonté de reconnaître la dette de Levinas par rapport à Heidegger : la Dichtung comme la littérature conduisent à la mise à l'écart du concept, impliquent l'une et l'autre une transcendance, une sortie hors de la métaphysique et du psychologisme. L'équivalence est là : si ce n'est, comme tu as raison de me le faire remarquer, que pour Levinas la pensée de l'être ne tient pas ses promesses, qu'elle reste une pensée du Même, et, comme je me suis risqué à le dire tout à l'heure à peine une pensée mais l'illusion transcendantale. En tout cas, la « langue qui parle » répond, fait écho à l'appel de l'Etre, tout en restant pour Levinas sans responsabilité. Mais c'est cette désacralité, cette désacralisation, propre à la littérature, qui, précisément me conduit à ne pas exclure l'expression " Belles lettres ». Là encore il s'agit d'une question de style : Heidegger, dans la sacralisation qu'il réalise de la Dichtung (on ne peut même pas traduire ce terme) évoque des textes rares et d'entente difficile, comme ceux de Hölderlin, Trakl, Rilke ou George, dont il donne des commentaires discutables et savants, au plus proches de sa lecture des présocratiques, tout cela en prétendant retrouver ce qu'il y a au fond de la langue antérieurement au désastre de la technique. Levinas au contraire va chercher la littérature la plus classique, la plus commune, 
celle qui était enseignée dès l'école primaire sous la troisième République (tout cela qui, aujourd'hui disparaît, entraîné dans le tourbillon de la mondialisation aux CARNETS DE CAPTIVITÉ), celle qui avait donné lieu à la France de l'affaire Dreyfus et à l'héroïsme de Zola. La France où l'on faisait rituellement à l'école des parallèles entre Corneille et Racine, où l'on apprenait «Booz endormi » («... et Ruth se demandait/ quel Dieu, quel moissonneur de l'éternel été/ avait en s'en allant négligemment jeté/ cette faucille d'or dans le champ des étoiles »), ou même celle d'un poète de distribution des prix comme Sully Prudhomme (« le coup dut l'effleurer à peine/ N'y touchez pas, il est brisé »: tout cela c'est les «Belles lettres ».

Fernanda Bernardo: J'en viens à la seconde question entraînée par ton propos précédent: ce que tu dis de "l'éveil » et de "la sortie de l'horreur de l'être " me conduit à revenir aux "Carnets de Captivité», très, très éclairants, à mon avis, par rapport à cette hypothèse (un peu bizarre, je trouve) de l'existence d'une première étape d'une pensée de l'être chez Levinas - ce qui ne veut pas dire, bien sûr, que rien ne s'est passé dans sa pensée entre, disons, DE L'ÉVASION (1935), LE TEMPS ET L'AUTRE (1947) et AUTREMENT QU'ÊTRE (1974) : le dessein, le rêve, le désir est pourtant toujours le même, ou il va toujours dans le même sens : celui d'aller au-delà du sens de l'être. Il s'agit toujours de sortir de l'être, de la brutalité et de l'horreur de l'être, et de la barbarie de la civilisation qu'il a tissée et qui l'accepte, afin de repenser et de renouveler la question de l'être en tant qu'être et, de la sorte, la philosophie même. Le style, le langage et les modalités de cette sortie ou de cette "excendance ", comme il la nomme dans DE L'ÉVASION, ont bien sûr changé, ils se sont raffinés - à mon avis en grande partie en réponse à la lecture de "Violence et métaphysique " (1964) de Jacques Derrida. Leur rencontre chiasmatique passe aussi par là, commence même peut-être là : par la manière de poser et de penser cette " excendance " - il faudra un jour le saisir en saisissant la différence entre la contraDiction (Levinas) et l'aporie (Derrida), notamment, ainsi que la portée, tout autre, de la radicalité, voire de l'hyper-radicalité, de la déconstruction derridienne par rapport à l'éthique levinassienne et au sens levinassien.

En effet j'avoue qu'un des très grands étonnements de ma lecture des CARNETS a été de découvrir le rôle du judaïsme comme condition ou incondition du Juif, et pas du tout comme doctrine, dans le frayage de cette nouvelle orientation de la philosophie. Et de découvrir que c'est justement le judaïsme - et en lui la matinalité de son enseignement du respect de l'autre - qui mène Levinas à se démarquer, enfin explicitement, de Heidegger. Ainsi, dans le Carnet 1 on peut lire : "refiguration juive 
opposée à la préfiguration grecque ou allégorie. Contre l'universalisme grec ». (p. 51). Et dans le "Carnet 7» : "Ma philosophie - est une philosophie du face-à-face. Relation avec autrui, sans intermédiaire. C'est cela le judaïsme. " (p. 186) Et dans le «Carnet 2» (p. 75) Levinas écrit très explicitement: "partir du DASEIN ou partir du J.)

Qu'en penses-tu? Et notamment, qu'en penses-tu par rapport à la question de la dépaganisation ou de la sainteté propre à l'éthique de Levinas, à même l'éthique au sens de Levinas, que tu as aussi précisé?

Alain David: Quant à la sortie de l'être, je suis pleinement d'accord avec toi : je ne pense pas qu'il y ait deux étapes, voire, j'y ai insisté, je suis d'avis qu'il y a, au-delà même de ce dont Levinas convient explicitement, une invalidation de l'idée d'une pensée de l'être. C'est là, à mes yeux, la conséquence de la notion de désacralisation (avec laquelle, je pense, tous les commentateurs qui veulent garder peu ou prou la référence à l'être ont des difficultés). Mais en contrepartie, à côté de cette inspiration profonde, qui a à voir avec le judaïsme (j'y reviens dans un instant) il y a sans doute une hésitation quant à Heidegger, à son rôle dans la phénoménologie : il a pu croire un temps, tout en hésitant à s'engager sur cette pente, que Heidegger allait plus loin, tirait des conséquences que Husserl ne voyait pas : l'idée d'une transcendance, à déchiffrer à même de l'élucidation infinie entreprise par la phénoménologie. Mais, je le répète, il a hésité. Dans son entretien avec Wolzogen il rappelle un mot de Scheler sur Heidegger : " c'est un mélange de génie et de prêchi-prêcha». Dès son séjour à Fribourg, il avait donc senti qu'il ne pouvait pas suivre Heidegger, même si, comme il le raconte avec la distance de l'humour, ce dernier passait (auprès d'un étudiant rencontré dans un train) pour " le plus grand philosophe du monde ». Le problème était alors pour lui d'aller effectivement plus loin, sans consentir au sacré. En ce sens il a trouvé, non dans "Violence et métaphysique », qu'il a ressenti comme une agression, mais dans La voix et le phénomène, qu'il considérait comme l'un des plus grands livres du XXème siècle, une possibilité essentielle. La proximité, le chiasme qu'il a évoqué et que tu rappelles, date de $L a$ voix et le phénomène. Derrida jugeait, comme tu le sais, que ce chiasme était «très effilé » : et en effet, plus je lis, plus j'ai le sentiment qu'entre Derrida et Levinas les oppositions ou les conflits sont de circonstances, et le compagnonnage très profond et infini. Et ce serait sans doute tout un travail, passionnant, que de pointer au-delà des différences de style, de références, de sensibilité, la manière dont une œuvre (je ne dis pas, je ne sais pas, laquelle) ne cesse de se reconnaître dans l'autre, de se laisser interpeller par elle, de la saluer. 
Quant au judaïsme, comme je le notais il y a un instant, je le vois là également : moins, comme une culture dont Levinas serait porteur (elle existe pourtant, très profondément chez lui, mais d'une certaine façon elle a varié, s'est transformée : ce n'est qu'après la guerre que Levinas, avec son maître Chouchani, est devenu un praticien du Talmud. Et depuis il n'a évoqué le judaïsme qu'à partir de la référence au Talmud) que comme une sensibilité, finalement philosophique. Le Judaïsme, dans le " pressentiment de l'horreur nazie », ce fut ce refus de la sacralisation, et l'attention portée à la littérature ; puis ce fut " le souvenir de l'horreur nazie », la manière dont la philosophie, si elle devait signifier une sagesse, devait mettre à l'abri de cette horreur. En cela même Heidegger (contrairement à Fink, écrit quelque part Levinas) a failli, n'évitant pas « de dire en grec des choses barbares ». Donc, oui, je suis là encore de ton avis : le judaïsme tient essentiellement, au-delà de ce qui serait en un sens plus étroit une culture juive, dans ce que tu nommes dépaganisation, c'est-à-dire dans le rejet du numineux, dans la désacralisation.

Fernanda Bernardo: Pour revenir encore à ta référence à la question $d u$ "noyau de la lecture " de Levinas que représente Husserl, et non sans (me) rappeler tout à la fois que Levinas disait que ce qu'il cherchait dans sa pensée était ce que Husserl lui-même appelait l'«originäre Gegebenheit», et que le tout premier mot du premier Cahier, daté du 8 septembre 1937 (bien avant, donc, la mobilisation et la captivité de Levinas!) est justement celui de "Phénoménologie », je voudrais te demander si tu vois quelque trace de la présence de Husserl, de la radicalité revendiquée par sa phénoménologie transcendantale notamment, ou bien alors par la condition d' "éternel débutant en philosophie ", l'éternelle jeunesse du philosophe, illustrée dans cette passion de Levinas pour la littérature, tellement attestée par les «Carnets»? Ou encore pour l'écriture dont le concept est pourtant si ambigu dans l'œuvre de Levinas.

Alain David: Tu touches là à quelque chose de très important à mes yeux, et qui, à mon sens, n'a pas été suffisamment relevé par les commentateurs : la référence à Husserl est certes régulièrement mentionnée, et les spécialistes de Husserl ne manquent pas en général de citer Levinas dans les bibliographies, et, çà et là de faire état de ses commentaires. Mais je relevais, tout à l'heure, la volonté de Levinas d'aller au-delà de Husserl, du point où ce dernier se serait arrêté, du sentiment éprouvé par le jeune Levinas que Husserl avait terminé son œuvre, qui ne réservait selon lui 
plus de surprises. Mais cela même est peut-être plus complexe, et il me semble qu'il faut continuer à réfléchir à la place occupée par Husserl dans l'élaboration de l'œuvre de Levinas. Ainsi, rangeant récemment ma bibliothèque j'ai mis la main sur un cahier datant du séminaire de 1976-1977, consacré d'une part à Michel Henry, au premier volume de L'essence de la manifestation, d'autre part à Expérience et jugement (ce dernier séminaire commencé en avril et poursuivi l'année suivante) : ce qui m'a remis en mémoire un épisode à cet égard significatif. Levinas m'avait attribué un exposé sur le $\S 21$,a, d'Expérience et jugement portant sur la négation. Il s'agissait d'une boule rouge et lisse par devant, verte et bosselée par derrière. J'étais désespéré, seul avec cette boule rouge, lisse, verte, bosselée, incapable de commenter autrement que par une insipide paraphrase. Je crois que cela a beaucoup amusé Levinas, il m'a confisqué la parole (selon son habitude lorsqu'il s'agissait de Husserl et que celui qui parlait était un étudiant), et s'est lancé dans un grand " numéro » sur la manière de Husserl, qui disait-il, ne « levait jamais le doigt pour attirer l'attention » (et à cet instant, je m'en souviens, Levinas était, dans une de ses attitudes familières, renversé sur son dossier, hilare et les deux index levés) et que tout le drame de la négation se ramenait chez lui à l'anecdote d'une boule rouge et lisse par devant, verte et bosselée par derrière.

Je me rappelle avoir encaissé cette leçon, un peu vexé, bluffé par la parole du maître, mais au fond n'ayant pas complètement compris ce qu'elle cachait, seulement confirmé dans mes sentiments d'intime hostilité envers Husserl. Réveillé depuis peu à ce souvenir je le comprends tout autrement : ce que Levinas mettait en évidence, à demi-mots, c'est le caractère absolument désacralisé, désacralisant, de la phénoménologie. Dans le passage que tu évoques, ce premier mot des Cahiers où il en appelle à la phénoménologie, il le fait en condamnant tous les énoncés qui oublient l'intentionnalité : " il y a de cela dans tel acte, il y a de ceci dans tel être. Comment ? Wie liegt es drin ? Pas même envisagé. » Or poser la question du comment, c'est au fond avoir la bonne attitude devant la magie. Dans tous les tours de magie il y a un truc. La phénoménologie dévoile les trucs, fait obstacle au merveilleux de pacotille, c'est-à-dire au sacré. La raison phénoménologique est en cela même l'accomplissement de la raison occidentale, elle ne laisse aucune place possible au sacré, fût-il ce sacré ultime qui surgit à proximité de ce qui passe pour la question philosophique par excellence : il y a, pourquoi y a-t-il quelque chose plutôt que rien ? Mais répond Levinas-avec-Husserl : wie liegt es drin ? La négation, ce n'est rien d'autre qu'un autre remplissement des attentes intentionnelles. Et les affects que l'on voudrait lui associer, l'émerveillement ou l'angoisse, la mort, le monde... ne sont qu'une mauvaise complaisance, parce qu'ils masquent finalement le véritable 
sens de la merveille : celui qui surgit en tant que langage comme l'un-pour-l'autre, l'autre-dans-son-expression. Cela aussi est le judaïsme.

Je ne voudrais pas m'étendre sur la question des nuances de Levinas quant à l'écriture : elle croise évidemment celle du rapport à Derrida à laquelle $\mathrm{j}$ 'ai déjà fait allusion, et mériterait à cet égard beaucoup d'attention, de prudence et de nuance. Et surtout, je le répète, il y a, me semble-t-il autre chose, qui autorise à aller vite ici : je suis convaincu que, quelles que soient les nuances nécessaires, Levinas a reconnu en Derrida une œuvre majeure, au plus proche de la sienne, peut-être l'œuvre qui lui était la plus proche. La réciproque me paraît vraie.

Fernanda Bernardo: Peux-tu alors préciser, encore davantage, en quel sens l'accent placé sur l'écriture de Levinas, si important selon toi dans les "Carnets», renouvelle la compréhension de la double référence à Heidegger et au judaïsme?

\begin{abstract}
Alain David: Bon, si tu m'autorises à simplifier, c'est-à-dire à esquiver le livre qu'il faudrait écrire ici, je dirai que l'écriture, au sens où on a compris ce terme avec Derrida, Blanchot, et d'une certaine manière Levinas, est absente chez Heidegger (même s'il me semble incontestable que Heidegger à sa manière fasse œuvre d'écrivain). Il n'y a par exemple pratiquement pas de place pour la littérature dans l' œuvre de ce dernier. Quel sens donner à cette opposition, à laquelle je reviens maintenant, entre Dichtung et littérature ? A mon avis - et je crois que cet avis se vérifierait aisément en entrant dans le concret des commentaires la Dichtung est cette parole célébrant la merveille de la donation. La littérature au contraire - mais il arrive à Levinas d'identifier l'œuvre de la littérature à celle de la poésie, par exemple dans l'article «Poésie et résurrection » - désacralise. Jamais la Dichtung au sens de Heidegger ne consentirait à l'incipit suivant : « longtemps je me suis couché de bonne heure »; ainsi André Breton refusant d'écrire : " la marquise sortit à cinq heures $»$. En revanche on pourrait revisiter les commentaires de Heidegger, aussi géniaux soient-ils, et constater comment, en un sens, Heidegger a détourné ou manqué ceux qu'il sollicitait, Trakl, et même Hölderlin. Ou ceux qu'il n'a pas osé solliciter, comme Celan. Faut-il parler en l'occurrence à nouveau de judaïsme ? La référence à Celan pousse à le faire, mais également celle à Blanchot, et bien sûr à Levinas lui-même. Permets-moi de me placer sous l'autorité d'une citation, la phrase, si choquante, si étrangère au climat heideggérien, de Marina Tsvetaeva : " tous les poètes sont des youpins ».
\end{abstract}


Fernanda Bernardo: Revenons aux surprises des "Carnets de Captivité», et à une surprise très touchante : nous rencontrons "Bobby ", le chien "Bobby » (p. 150) dans les "Carnets»... On dirait qu'il est là, ce chien tellement touchant, comme un exemple, j'ose le dire, de l'éthicien selon Levinas, puisqu'il écrit que celui-ci aimait les prisonniers "sans arrière-pensée, en dehors de toutes nos distinctions et règles sociales.»

Alain David: Eh oui, il y a ce témoignage. C'est peut-être celui qui de tous m'interpelle le plus. Je pense qu'on a ici un exemple, peut-être l'exemple le plus frappant, le plus convaincant, de la transformation littéraire à laquelle en appellent ces " Carnets de captivité ». Dans « Nom d'un chien ou le droit naturel » l'anecdote émouvante (ce qu'on pourrait prendre comme tel) est devenue la matière d'un récit extrêmement élaboré. Il faudrait lire ligne à ligne ce texte, s'y arrêter longuement. Faute de pouvoir le faire ici je dirai seulement que Bobby devient un personnage clé, " ami de l'homme », c'est-à-dire sujet par excellence non ouvert au monde, et comme tel désacralisé, porteur en cela de sainteté, attestant d'un langage tout entier expression et non plus logos : " pure nature ouverte sur des droits », « transcendance ouverte dans l'animal », « dernier kantien de l'Allemagne nazie, n'ayant pas le cerveau pour exprimer la maxime de ses pulsions » "son aboiement d'ami, foi d'animal, naquit dans le silence de ses aïeux des bords du Nil ». On a là, au plus proche de Derrida, encore une fois, une lecture d'une audace incroyable, l'élément d'une déconstruction radicale de la phénoménologie, de la philosophie.

Fernanda Bernardo: Oui, on a là une déconstruction de la tradition $d u$ «zoon logon ekhon» aristotélien. Mais je crois qu'il y a là aussi une marque de la limite même de l'éthique levinassienne - et au sens de Levinas - par rapport à la déconstruction derridienne. Celle qui va du tout autre, propre à l'éthique d'Emmanuel Levinas, au tout autre est tout autre, propre à la déconstruction de Derrida : une limite qui répond finalement à la question : qui est l'autre, le tout autre, chez Levinas et chez Derrida? Et une limite qui à mon avis révèle l'humanisme profond, bien qu'autrement repensé, certes, de l'éthique levinassienne qui - on a tellement du mal à le croire! - ne se sent pas encore regardée par "l'animal » qui, pour Levinas, n'est pas un visage ni même un "tiers». Un passage assez troublant au Carnet 4 semble confirmer ce registre anthropocentrique de la pensée de Levinas qui, tel que Derrida le remarque, finit par inscrire son éthique de la responsabilité infinie dans la tradition subjectale (et sacrificialiste) de la philosophie occidentale. En effet, il y écrit : "Mon 
regard ne s'élève qu'au niveau où il [...] peut rencontrer un être humain. Je ne vois pas ce qui est à mes pieds. ") (p. 113)

Alain David: Tu poses à nouveau une question difficile : jusqu'où va le compagnonnage de Levinas et de Derrida, et ce chiasme effilé dont parlait Derrida, jusqu'à quel point est-il effilé ? Et si j'entends bien ta question, dans quelle mesure Derrida va-t-il plus loin que Levinas ? La réponse qui spontanément me vient est que je ne sais pas. On peut sûrement découvrir chez Levinas un anthropocentrisme impénitent et non surmonté, et spontanément, dans la conversation, dans son idiosyncrasie, c'est peut-être ce qu'il était, petit bourgeois, humaniste, anthropocentrique... Mais inversement, au moment où on lui demanderait : " mais que faites-vous de l'animal, qui n'appartient pas à la même espèce - j'ai failli dire : à la même race - que nous ", ne consentirait-on pas à ce qui précisément est en cause au fond de l'anthropocentrisme, l'inscription de la vérité dans une forme, c'est-à-dire, dans les termes de Husserl, un naturalisme. On reprocherait alors à Levinas l'étroitesse du sien, à la manière de ces écologistes qui disent : «mais l'homme n'est pas la seule espèce sur la terre, il y en a d'autres. » Or, selon ce que je comprends, le texte que tu cites des Carnets est d'abord une contestation du naturalisme : il dit que voir n'est pas un fait biologique. Je vois, seulement là où il y a ouverture. Un être qui serait « naturel » ne verrait pas. Et dans leur naturalisme, les hommes ne voient pas (« comme si le regard naïf, dans son intention ontique de regard, se trouvait bouché par son objet » dit Levinas dans « La philosophie et l'éveil »). L'ouverture, c'est 1'humanité. Mais cette référence à l'humanité légitime-t-elle l'objection : «que faites-vous de l'animal ? » Je répète que cette question peut être, à mon avis, retournée à ceux qui la posent, en leur demandant : «comment sortez-vous du naturalisme ? » Car sortir du naturalisme et « étendre à l'infini le domaine de la lutte » c'est engager un langage dont je ne vois pas comment il ne serait pas celui de l'humanisme : mais de l'humanisme de l'autre homme. Car « l'autre homme » n'est pas enfermé dans une nature humaine, il peut, si je lis correctement « Nom d'un chien ou le droit naturel » avoir la petite figure poilue de mon chat, de l'animal familier, offert sans réserve à ma violence, et envers qui, « je ne peux plus pouvoir » : qui est donc, par excellence, un visage. L'humanité de "l'autre homme » n'est pas la nature humaine. Elle est à la démesure du commandement : si l'infini n'a ni bornes ni limites, ce n'est pas au sens de la curiosité de l'enquête naturaliste mais au sens du commandement, qui en cela est le registre même de l'épochè. C'est pourquoi, pour ma part, je ne vois pas de différence entre le «tout autre » de Levinas et le « tout autre est tout 
autre » de Derrida (à moins, encore une fois, de vouloir demeurer dans un naturalisme, ce qui ne serait ni Derrida, ni Levinas). Ce que Levinas et Derrida ont en commun, comme l'avait pensé Levinas lui-même, c'est la radicalité de la réduction.

Fernanda Bernardo: Oui, et le «tout autre» de Derrida est «quiconque», «n'importe qui» et pas seulement "l'autre homme», le prochain ou le semblable... Derrida remarquant souvent que sa différance concerne différentiellement tous les vivants et tous les rapports du vivant au non-vivant...

Mais je reviens finalement à ce qui fut ma surprise dans ma lecture des «Carnets de Captivité» et donc à ce dont, à mon avis, ils portent le témoignage par excellence, à savoir, celui de la réponse (le «me voici», hinneni) d'Emmanuel Lévinas à l'expérience et par l'expérience de sa captivité. En effet, ma surprise ne fut surtout pas dans le souci de Levinas d'une ouvre à faire sous le triple registre de la philosophie, de la littérature et de la critique. Ma surprise fut surtout celle de découvrir l'importance de l'expérience de la captivité - vécue, qu'elle le fut, à la fois comme une expérience et de l'être, de l'horreur de l'être, et du judaïsme - pour le frayage de la voie que Levinas se cherchait, au moins depuis 1935, depuis DE L'ÉVASION, notamment, pour sortir de l'être et pour marquer son opposition explicite, pour la première fois explicite envers Heidegger. C'est justement cette opposition pour la première fois explicite à Heidegger - si l'on fait attention au parcours philosophique de Levinas jusqu'à sa captivité - que se marque et se donne à lire dans les «Carnets» par le biais de l'expérience de la captivité singulièrement vécue comme une expérience du judaïsme. En effet, dans le "Carnet 2», daté de 1942, Levinas écrit : "Partir du DASEIN ou partir du J.». Et dans le «Carnet 5» il ajoute: "Un élément essentiel de ma philosophie - ce par quoi elle diffère de la philo. de Heidegger - c'est l'importance de l'Autre.». Et au "Carnet 7» Levinas précise : "Dans la persécution je retrouve le sens originel du J.» Et encore, et toujours au «Carnet 7» : «Ma philosophie - est une philosophie du face-à-face. Relation avec autrui, sans intermédiaire. C'est cela le judaïsme.»

Dans la scène d'une pensée à la recherche de son timbre propre, comme celle des "Carnets», on a l'impression qu'outre les quasi-concepts de "persécution " et d' "otage ", par lesquels, à partir de la deuxième moitié des années 60, Levinas pensera ce qu'il appelle l'incondition de la subjectivité du sujet - Levinas ayant même avoué à Michaël de Saint-Chéron que ce mot d'«otage», il le connaissait depuis la persécution nazie -, on a donc l'impression, disais-je, que la souffrance de "l'expérience 
de la captivité» aura non seulement inspirée à Levinas le timbre méta-éthique de sa pensée - timbre par lequel, contre le tout de l'occidentalité philosophique, il fera de l'éthique la philosophie première -, mais aussi la passion absolue de la récurrence de l'ipséité qu'il nous donnera à penser dans AUTREMENT QU'ÊTRE, notamment.

Alain David: Quelle est la résonance de la captivité dans la pensée de Levinas? Tu as raison, le vocabulaire même de cette pensée - tous les termes que tu relèves, et d'autres encore sui generis - renvoie à cette expérience. En même temps je suis frappé à la lecture des Carnets, par le peu de place réservé au vécu quotidien dans le camp. Nous n'avons pas affaire à un journal tenu au jour le jour, on n'est pas non plus dans le registre tragique que prendra un texte comme "Sans nom » par exemple. Il ne me semble pas, en ce sens, que la pensée de Levinas soit la traduction de son expérience personnelle, à moins de dire que cette expérience personnelle est une expérience enrichie : enrichie, dans le double registre d'une hyperbole de la rétention et de la protention, de toutes l'anticipation que portaient les lectures - la littérature comme le préphilosophique - de toute la mémoire ensuite associée aux témoignages, faisant sien, dans la logique de la substitution, tout ce qui n'a pas été vécu en première personne, et conduisant à l'exergue d'Autrement qu'être, «A la mémoire des êtres les plus proches parmi les six millions d'assassinés par les nationaux-socialistes, à côté des millions et des millions d'humains de toutes confessions et de toutes nations, victimes de la même haine de l'autre homme, du même antisémitisme. » Là, je suis d'accord : la surprise, c'est bien le rôle de l'expérience dans la pensée d'Emmanuel Levinas, et l'exergue d'Autrement qu'être donne la mesure, la démesure, de cette expérience.

Fernanda Bernardo: Je partage ta surprise du fait que Levinas ne parle pas de son expérience personnelle de prisonnier dans les «Carnets de captivité» - à l'exception près de deux ou trois très brefs passages... Ce ne sera que dans la deuxième partie de ce premier volume des Euvres complètes, qui intègre les "Ecrits sur la captivité et Hommage à Bergson » (p. 199-219) rédigés peu après son retour de captivité, que Levinas parlera soit de la "captivité 》 soit de sa propre "expérience de la captivité ». Mais là encore de sa propre expérience de la captivité comme de celle d'un autre, car, et pour l'essentiel, il n'en parlera que par l'évocation et par la réflexion sui generis de la souffrance du "prisonnier juif » (qu'il fut, lui-même, aussi) dans les stalags et les oflags. Comment écoutes-tu ce silence? Cette discontinuité, cette discrétion. 
Alain David: C'est une question difficile : plus que difficile, délicate, parce qu'elle me semble impliquer la pudeur, et qu'il serait inconvenant de faire montre de moins de pudeur que n'en avait eue Levinas lui-même. Ceci posé, il me semble que la réponse, qui supposerait, selon ta propre expression, une écoute du silence, peut aller dans plusieurs directions. Il y a d'abord ce que je suggérais il y a un instant : la volonté littéraire, le caractère littérairement surinvesti de l'expérience selon Levinas. En ce sens ce qu'on peut savoir des esquisses de roman, aussi inaboutis que soient ceux-ci, est significatif : la captivité se trouve chez Levinas au confluent de la littérature et de la philosophie. Peut-être faudrait-il dire : la philosophie comme littérature. Ou la littérature comme pensée.

Mais, puisque tu poses la question, j'oserai deux autres hypothèses qui, comme on dit, n'engagent véritablement que moi. Il y a déjà la réserve - je disais la pudeur - constamment manifestée par Levinas vis-à-vis de la déportation : le fait que lui-même a eu le sentiment d'être un privilégié, parce qu'il n'avait pas connu les camps de concentration ou d'extermination : il parle quelque part de «l'injuste privilège d'avoir survécu », il parle ailleurs d'une "tumeur de la mémoire »: or une tumeur empêche le bon fonctionnement de l'organe affecté. Il n'y a pas véritablement de mémoire de l'extermination, de mémoire de la souffrance juive. Et, quoi qu'il en soit, il n'y en a pas une " mémoire volontaire » pour reprendre l'expression célèbre de Proust, dont tu n'ignores pas que Levinas le lisait beaucoup et sur qui il avait écrit dès le lendemain de la guerre. Mais au-delà de cette hypothèse je ferai intervenir un phénomène constant et souvent observé : l'étonnant silence des témoins, au sortir de la guerre. Il me semble qu'il faut, à ce sujet, aller plus loin que l'explication souvent invoquée : " personne ne nous aurait crus ». Il me semble, pour en avoir ressenti moi-même les effets très petit (je suis né immédiatement après la guerre) qu'il y avait à ce moment une hésitation de la part des Juifs à se réclamer du fait juif, comme si quelque chose de l'antisémitisme d'avant guerre fonctionnait encore dans la société d'après-guerre, comme si il était licite et glorieux d'invoquer la déportation pour faits de résistance, mais que la déportation liée à l'antisémitisme était en quelque sorte inavouable. Comme si les Juifs après la guerre n'avaient aspiré qu'à une seule chose, retrouver au sein de la société française un anonymat tranquille, avec au mieux la possibilité de vivre leur condition religieuse d' "Israélites ». Tu te rappelles la dernière phrase du Procès de Kafka : " et c'était comme si la honte dût lui survivre ». La honte survivait, rémanente à l'intérieur de la société. Et Levinas, et plus tard Derrida, ont été de ceux qui ont permis de voir dans le judaïsme non plus une particularité douteuse et ambiguë, et finalement honteuse, mais une catégorie de pensée, l'un des phénomènes les plus complexes et les plus 
intéressants de la vie occidentale et de la pensée philosophique. Mais à l'époque des Carnets cela n'était sans doute pas si net, même pour Levinas. S'il faut citer un exemple, permets-moi d'invoquer le cas de Bergson, justement parce qu'il est cité et admiré par Levinas : car, quoi qu'il en soit de son attitude admirable, il avait eu dans son testament des mots qui attestaient eux aussi de l'atmosphère d'une époque - mots censurés dans l'édition du Centenaire par la grâce de son œuvre: "Je me serais converti si je n'avais vu se préparer depuis des années (en grande partie, hélas ! par la faute d'un certain nombre de Juifs entièrement dépourvus de sens moral) la formidable vague d'antisémitisme qui va déferler sur le monde. " (cité par Philippe Soulez, dans Bergson politique, Puf 1989 p. 326). Je ne veux pas, en citant la parenthèse du testament (que Levinas ignorait certainement) faire un procès dérisoire à Bergson, dont le courage, au moment où il était le plus difficile d'en avoir, fut exceptionnel : mais en rappelant ce propos, ô combien gênant, je voudrais essayer de faire valoir le contexte d'une époque qu'il faut juger, certainement, mais qu'on ne peut complètement comprendre avec les yeux d'aujourd'hui. En d'autres termes, il me semble que dans les année 40 le regard porté sur le fait juif n'était pas, même pour Levinas, celui qui a valu ensuite, et qui, je me plais à le redire, a changé notamment par la grâce de son œuvre. On m'objectera que ces explications sont aventurées et qu'elles se contredisent, qu'il faut choisir entre les trois. Je ne le crois pas, elles ne sont pas sur le même plan, la troisième est circonstancielle, la deuxième fait intervenir le rapport qui a été celui de Levinas à un événement impossible. La première rejoint l'œuvre, considérée dans sa plus profonde portée.

Fernanda Bernardo: Permets-moi de revenir encore sur un point qui, en dépit de ce que tu as pu dire tout à l'heure, me laisse encore perplexe: le judaïsme. Nous savons qu'un des motifs du débat de Derrida avec Levinas, de leur rapport chiasmatique effilé est bien celui de "l'exemplarisme juif», avec chez Levinas la conviction du caractère exemplaire de la condition, ou comme il dit, de l'incondition juive. Le «me voici» (hinneni), le «je suis juif» résonnant chez lui comme l'accusatif d'une réponse hétéronomique à l'injonction de l'autre homme dont il est otage. Et, on le sait, Levinas fait de la singularité de cette dissymétrie hétéronomique le trait universel de l'humain. L'incondition de la "spiritualité» juive étant donc celle de l'humain - de l'humain humain. Jacques Derrida au contraire tient que quiconque répond à un appel, à un appel de l'autre (un autre - je le rappelais tout à l'heure - qui chez lui se trouve être quiconque parce que " tout autre est tout autre», justement) devra cependant toujours continuer à douter, à se demander s'il a bien entendu, s'il n'y a pas de malentendu 
originaire, bref, s'il est bien le premier destinataire de cet appel. C'est immense... Et cela fait trembler toute notre civilisation abrahamique... en lui donnant pourtant, en la rappelant pourtant à son à venir. L'hyper-radicalité et la singularité de la déconstruction derridienne passe par là... Ainsi que la condition de "marrane universel» (de celui qui reste fidèle à un secret qu'il n'a pas choisi) de Jacques Derrida, le "premier»/ ou le «dernier des juifs».

Cela dit, ma question est alors la suivante : dans quelle mesure crois-tu que l'épreuve de la captivité aura été décisive pour cette détermination de l'exemplarité universelle du Juif chez Emmanuel Levinas (selon, bien sûr ce qu'on peut en savoir). Ne penses-tu pas, Alain, que cette épreuve, outre le fait qu'elle éclaire la genèse de l'idiome méta-éthique de Levinas dans sa démarcation profondément critique avec Heidegger (et pour la première fois explicitement critique) et l'ontologisme de la philosophie occidentale, permet également de mieux comprendre ce que je viens de nommer dans la trace de Derrida sa "tentation exemplariste " (le caractère exemplaire du judaïsme). Je voudrais rappeler pour finir, cette annotation du "Carnet 7»: "Dans la persécution, je retrouve le sens originel du J., son émotion initiale. Non pas persécution quelconque persécution absolue, qui pourchasse l'être de partout [...] situation du "subir " pur où il y a une élection [...] le subir devient ici : filialité."

Alain David: Je voudrais essayer de résister à tes hypothèses, et ce n'est pas facile, parce qu'à certains égards, à beaucoup d'égards sans doute même, je te donne raison. La citation que tu fais des «Carnets» semble aller absolument dans le sens que tu suggères. Levinas semble le reconnaître - il le reconnaît, je l'admets - il y a une condition exemplaire du Juif. Mais si je te suis jusqu'au bout, on aurait la situation suivante : Levinas, partant de son expérience de la persécution pour élever son cas particulier, et la réalité des Juifs face à la persécution, au rang de paradigme, tandis que Derrida n'accorderait pas au judaïsme ce redoutable et discutable privilège : tout autre est tout autre, et, donc, pas nécessairement un Juif. Je suis convaincu, comme toi sans doute, qu'il y a chez Derrida cette critique de Levinas. Et je viens de l'admettre, je crois qu'on peut trouver matière chez Levinas à l'étayer. Mais jusqu'à quel point peut-on tenir sur cette ligne : car l'inverse existe aussi. Il me semble que la dédicace que j'ai citée tout à l'heure, d'Autrement qu'être, est absolument claire : si l'antisémitisme est bien la persécution qui touche les Juifs c'est cette persécution qui constitue - phénoménologiquement, si on peut dire ainsi - la condition juive, et non l'inverse (en ce sens il y a, en compliquant l'argument, certes, un hommage au Sartre des Réflexions sur la question 
juive). Si j'osais pasticher Derrida je dirais «tout autre, persécuté (et l'autre n'est-il pas, selon son altérité même, persécuté ?) est tout autre, juif. » Mais je pourrais citer encore bien des textes, voire bien des faits qui vont dans ce sens : ne serait-ce que l'irritation (le mot est faible) de Levinas lorsqu'on voulait faire de lui un "philosophe juif ».

Tu relèves encore que les Juifs sont les " otages. " Je vois là un malentendu : dire que je suis l'otage de l'autre ce n'est pas décrire la situation des Juifs face à la persécution nazie, c'est au contraire me placer face à la faiblesse d'autrui, à la force paradoxale de sa faiblesse qui fait que je ne peux plus vouloir. Un lien se noue que je ne peux défaire sans violence. Autrui m'obsède. Le « me voici» me met en posture de coupable - le fameux «nous sommes tous coupables, et moi plus que tous les autres » de Dostoïevski (qui n'est pas, puis-je le souligner, un « auteur juif »). C'est comme cela que je comprends le terme d'otage. Mais ce qui te trouble encore est que Levinas met les Juifs en première personne, du singulier ou du pluriel, se réclame de la condition juive pour parler, là où Derrida introduirait un doute fondamental. Pourtant, ce doute n'existe-t-il pas également chez Levinas. Je le cite : «Le dérangement dérange l'ordre sans le troubler sérieusement. Il y entre de façon si subtile qu'il s'en est déjà retiré, à moins que nous le retenions. Il s'insinue - se retire avant d'entrer. Il ne reste que pour celui qui veut bien lui donner suite. Sinon il a déjà restitué l'ordre qu'il troublait : on a sonné et il n'y a personne à la porte : a-t-on sonné ? " (En découvrant l'existence... p. 290) Non, vois-tu, je persiste à penser (un peu comme Leibniz qui disait que les philosophes ont tort dans ce qu'ils nient et raison dans ce qu'ils affirment) qu'à bien regarder, à y regarder par deux fois, au-delà des premières déclarations et des premières évidences, le chiasme entre Levinas et Derrida est bien plus effilé qu'il n'y paraît d'abord, qu'il ne faut pas se dépêcher de trancher entre les deux, ne serait-ce que parce que les positions de l'un et de l'autre se ramènent à une expérience philosophique, une expérience qui mobilise de façon puissante et géniale toute l'histoire de la philosophie et non seulement une expérience vécue, quelle qu'ait été la prégnance de cette expérience dans l'élaboration d'un certain idiome; que l'un et l'autre, donc, à travers un idiome effectivement lié à leur expérience la plus particulière, ont le génie de pouvoir faire entendre une affirmation sans limite. Tu m'objectes l'idiome, la particularité de tout idiome, en l'occurrence la référence à l'expérience particulière du judaïsme, au « je suis juif ». Mais qui ne dirait pas, pour peu qu'il en ait le courage, face à la persécution, " je suis juif »? Les étudiants de 68 en étaient venus à prendre pour slogan de manifestation «nous sommes tous des Juifs allemands. » Au-delà de ce slogan (qui émouvait 
Blanchot, qui y voyait cependant une incompréhension du fait juif) les choses sont néanmoins plus compliquées. Car posons-nous, toi et moi, la fameuse question : qui est juif ? Posons-nous la à propos de nous-mêmes. Suis-je juif ? Es-tu juive ? Oui, non, peut-être, cela dépend... Au-delà du oui et du non que chacun, selon son histoire familiale, peut faire valoir, aucune réponse n'est décidable. Jusqu'à quel point le suis-je, jusqu'à quel point ne l'es-tu pas ? Il m'est arrivé d'écrire que (en référence à l'existence selon Kant) « juif » n'est pas un prédicat - au sens littéral de prae-dicatum, ce qui est dit à propos d'un sujet. Et il m'arrive souvent, quand ce sujet est abordé de citer Joyce, dans le passage où, dans Ulysse, il met en perspective les relations de Bloom et de Stephen. Qu'est-ce que Bloom pensait de Stephen ? La réponse, extravagante et alambiquée, est la suivante : " Il pensait qu'il pensait qu'il était juif, tandis qu'il savait qu'il savait qu'il savait qu'il ne l'était pas ». Autrement dit Bloom pensait que Stephen pensait que Bloom était juif, tandis que Bloom savait que Stephen savait que Bloom savait que Stephen n'était pas juif. Le savoir porte sur le non judaïsme, tout savoir est un savoir de savoir et se partage, dans l'universalité (ce que Bloom sait, Stephen le sait également et Bloom sait que Stephen sait qu'il le sait.) Le judaïsme, lui, est une présomption, voire une présomption de présomption : il affecte néanmoins, en tant que présomption justement, le ou les savoirs. C'est selon un tel registre, peut-être le registre par excellence de l'indécidable, que je crois Derrida au plus proche de Levinas. Reste alors l'idiome - méta-éthique, juif, anthropologique dis-tu - de Levinas : oui, il y a cet idiome, mais tout idiome rentre dans une écriture, qui pense plus loin que l'idiome lui-même, et nous retrouvons à cet égard, à nouveau, l'effilement du chiasme.

Fernanda Bernardo: L'approche de "l'effilement» de leur rapport chiasmatique reste sans doute un grand travail encore à venir. Et qui passera, oui, sans doute, par l'intraductibilité de leur idiome.

Dis-moi, étant donné ta passion pour la phénoménologie, comment est-ce que tu envisages le tournant méta-éthique que Levinas a fait subir à la phénoménologie (et à la FUNDAMENTALONTOLOGIE) que d'ailleurs il a introduite en France dans les années 30 ? Introduction par laquelle ou dans laquelle, tout en respectant la voix de l'autre - ou plutôt de ces autres que furent pour lui Husserl et Heidegger, ses Grecs modernes -, il fait manifestement déjà aussi résonner son timbre à lui : un timbre où se marque soit la résonance anthropologique et humaniste de sa pensée soit ce qu'il tient par la vocation même de la philosophie. 
Alain David: Pour le dire le plus simplement et rapidement (on pourrait, sur cette seule question, écrire plusieurs livres) je dirai qu'il n'y a pas, selon moi, véritablement de tournant pour Levinas. Je ne crois pas qu'il se soit dirigé vers la phénoménologie pour à un moment donné lui faire violence, forcer le passage et lui faire prendre un tournant. Je pense qu'il a d'emblée retenu d'elle ce qu'il avait perçu ailleurs sur le mode du pressentiment, comme je le disais tout à l'heure, sur le mode du pré-philosophique, avec la littérature. Tu sais, je pense vraiment que d'un bout à l'autre de sa pensée, il est resté fidèle à cela : la philosophie a pour tâche d'exprimer quelque chose qui est présent dans les livres l'une de ses phrases les plus importantes me semble être celle-ci : «plus intime que l'intimité, c'est un livre ». Or la phénoménologie pour lui, c'est cela, le retour aux choses mêmes, au concret, l'attention accordée au comment intentionnel. Or, justement, la littérature raconte des histoires parce qu'elle ne dit pas ce qui est là, mais le comment de la vie, elle est à cet égard, comme l'écrit Proust (que je cite chaque fois que je le peux) «la vie, la vraie vie, la vie enfin vécue ». C'est de cela que Levinas s'empare, au titre de l'intentionnalité (« la phénoménologie, c'est l'intentionnalité ! »), au titre aussi de la réduction, le sujet qui diffère du monde, qui en diffère absolument (et c'est ce qui est pour la littérature la fiction) A cet égard encore le sujet n'est pas un upokeimenon, un $Z u$ -Grunde-liegendes (comme Rudolf Boehm le reprend de Heidegger). Il se dégage de ce contexte mondain et héllénique pour s'intriquer dans l'intrigue (littéraire) de l'infini. Husserl et Heidegger ne sont donc pas simplement des Grecs, ils ont, à mon sens, en inventant la phénoménologie, pour Levinas pressenti et ouvert cette voie, ils ont permis de se dégager du psychologisme (en particulier le Heidegger de Sein und Zeit, avec la transcendance ontologique), et j'ajouterai même qu'ils lui ont permis de se dégager de l'anthropologisme, si ce dernier signifie la place prégnante de l'homme dans le monde. Levinas n'a aucune difficulté par exemple à consentir aux thèses du structuralisme, parce que l'homme qui est mis en cause l'est également par lui. La transcendance de l'homme qui selon lui oriente la pensée (cf L'humanisme de l'autre homme) enlève l'homme à l'immanence du monde et engage une pensée " du dehors ", le visage ne présuppose aucun préalable quant à un a priori qui serait la figure de l'Homme, effacée dans la modernité comme, explique Foucault, par la mer un visage de sable.

Fernanda Bernardo: Rappelons, si tu le veux bien, pour les lecteurs portugais, l'épisode de la tasse de thé, que tu as vécu chez Emmanuel Levinas - quelle est aujourd'hui en toi la résonance de cet épisode? 
Comment l'envisages-tu, comment te parle-t-il aujourd'hui de l'homme et du philosophe Levinas?

Alain David: Je suis content que tu évoques cette anecdote, et que nous terminions par elle: elle me semble en effet riche et significative, et on n'a peut-être pas accordé suffisamment d'importance à ce qu'elle indique. Je la raconte rapidement: un après-midi j'étais en visite chez Levinas, comme cela m'arrivait deux ou trois fois par an. Il m'offre le thé (souvent, à mon avis pour s'autoriser à en prendre lui aussi, il m'offrait du Cointreau, une liqueur à base d'orange: «ça mord agréablement... ») Arrive le kinésithérapeute de Mme Levinas. Se levant pour saluer, Levinas me propose: vous voulez encore du thé, moi je ne prends qu'une tasse, je suis monothéiste», faisant mine ensuite de s'excuser, comme on le fait d'une plaisanterie douteuse, avec un bon sourire, après que le kinésithérapeute se soit retiré avec Mme Levinas: «c'est une blague pour kinésithérapeute ». Je te disais que j'accordais personnellement beaucoup d'importance à cette anecdote: elle traduit déjà quelque chose de constant chez Levinas, une sorte d'humour bienveillant. Cet humour le conduisait à se mettre en cause lui-même, y compris sur les choses sacrées - surtout même sur les choses sacrées. A se remettre en cause en allant chercher non le savoir philosophique mais la langue commune: j'ai insisté tout à l'heure sur son attirance pour ce qu'il appelait « les pages roses du Larousse » - ici la langue «pour kinésithérapeute» - ce qui ne traduit évidemment ni du mépris social ni une quelconque condescendance, mais l'attirance pour ce fonds littéraire présent dans la langue la plus quotidienne, cette capacité pour celle-ci de laisser résonner le «plus inscrit dans le moins», l'attention portée à ce que Derrida appelle le «tout autre»-si tu veux, Dieu, ou le Messie, ou la sainteté, arrivent aussi selon l'occasion et le visage de celui qui vient de sonner, impromptu, le premier venu, et qui est, là, le kinésithérapeute. Cette façon de désacraliser - désacralisation de tout, des institutions, de la religion, de l'autorité, de sa propre posture de sujet - est finalement pour Levinas le judaïsme dans ce qu'il a de plus vivant, le fameux humour juif. Levinas m'avait ainsi raconté une anecdote qui a été également publiée. Un ami israélien lui rend visite. Il voit dans l'entrée de son appartement, dans un rayonnage au-dessus de la porte, l'œuvre complète, en russe, de Pouchkine et s'exclame : «Ah ! je vois qu'on est dans une maison juive ». N'est-ce pas une belle définition du judaïsme, entendu dans l'inexistence qui est la sienne, dans sa récurrence et son insistance prodigieuse au sein de cette inexistence? 